
\title{
Reseacch Soute \\ Information-Sharing Reduces Anxiety Towards Vaccines for SARS-CoV-2 in Patients With Autoimmune Liver Disease
}

\author{
Iwate Medical University \\ Yuji Suzuki \\ Iwate Medical University \\ Hiroaki Abe \\ Iwate Medical University \\ Hisashi Eto \\ Iwate Medical University \\ Kenji Yusa \\ Iwate Medical University \\ Yasuhiro Takikawa \\ Iwate Medical University
}

Keisuke Kakisaka ( $\square$ keikaki@iwate-med.ac.jp )

\section{Research Article}

Keywords: COVID-19, Vaccination, Information-sharing, Autoimmune liver disease

Posted Date: September 24th, 2021

DOI: https://doi.org/10.21203/rs.3.rs-916240/v1

License: (c) (i) This work is licensed under a Creative Commons Attribution 4.0 International License.

Read Full License 


\section{Abstract}

Background: Patients with autoimmune liver disease may feel anxiety towards vaccination for Severe Acute respiratory syndrome coronavirus-2 because of insufficient data regarding its efficacy and adverse effects. The aim of this study was to study how information-sharing regarding coronavirus disease 19 (COVID-19) affects anxiety towards the vaccine in patients with autoimmune liver disease.

Methods: Information about COVID-19 and its vaccines provided to subjects with autoimmune liver disease, and change of anxiety to the vaccines after information-sharing were prospectively evaluated.

Results: Seventy-one patients prospectively and consecutively registered between March 2021 and May 2021 were assessed. Information-sharing decreased patient anxiety scores towards vaccination from 46 to 23 ( $p$ < 0.01). A beneficial effect of information-sharing was confirmed via a visual analog scale (VAS). The correlation of VAS before information-sharing to change of VAS during the study was negative (rho = $-0.405, p=0.00046)$. There was no significant difference regarding the comparison of VAS to age, or medication use.

Conclusion: Information-sharing improved anxiety towards vaccination in patients with autoimmune liver disease, especially in patients who felt strong anxiety before information-sharing.

\section{Introduction}

Coronavirus disease 2019 (COVID-19) was first reported in Wuhan, China, in December 2019 and later spread worldwide ${ }^{1}$. In Japan, the first vaccine for Severe Acute respiratory syndrome coronavirus-2 (SARS-CoV-2) was approved on February $2021^{2}$. Immunization is thought to stabilize the public health system by decreasing the number of patients who require intensive care ${ }^{3}$. Thus, the government has provided the chance to receive the vaccine for SARS-CoV-2 to all citizens if the citizens have no contraindication ${ }^{4}$. Although exactly which members of the population receive beneficial effects from vaccination against COVID-19 has not been established, citizens must opt-in to receive the vaccine. Because preexisting disease is a factor associated with unfavorable prognosis in patients with COVID$19^{1}$, patients with chronic diseases require explanations with updated information about the pros and cons of vaccination to SARS-CoV-2 to aid their decision. Despite a lack of data that vaccination affects the clinical course of COVID-19 in patients with chronic disease, most patients with chronic disease choose to receive the vaccine ${ }^{5-7}$.

As aforementioned, passive immunization using vaccines is essential for controlling disease spread ${ }^{8}$. Thus, increasing the number of people who receive SARS-CoV-2 vaccines without hesitation is crucial for public health. However, some have reportedly refused vaccinations due to fear of their side effects and insufficient data about these recently established vaccines for SARS-CoV-2 ${ }^{9,10}$. Patients with chronic disease, in particular, may experience intense anxiety in this regard. To perform vaccination universally, it 
is crucial to know how many patients with chronic diseases feel anxiety about the vaccine and understand how the anxiety can be reduced.

Autoimmune liver diseases, such as autoimmune hepatitis $(\mathrm{AlH})$, primary biliary cholangitis $(\mathrm{PBC})$, primary sclerosing cholangitis (PSC), and overlap syndrome may progress to liver cirrhosis and these autoimmune liver diseases, except for PSC, may require administration of immune-suppressive agents to control disease activity ${ }^{11-13}$. Because the disease severity and medications of patients with autoimmune liver disease would be affected by COVID-19 severity and/or response to the vaccine ${ }^{14}$, patients with autoimmune liver diseases require adequate information about vaccination to make informed decisions. However, whether patients with autoimmune liver disease feel anxious about the vaccine for SARS-CoV-2 and, if so, how this anxiety can be reduced remains unclear. The primary aim of this study was to determine whether adequate information-sharing about COVID-19 and vaccination in individuals with autoimmune liver disease could decrease anxiety toward the SARS-CoV-2 vaccine, resulting in increased support for decisions regarding vaccination for SARS-CoV-2.

\section{Methods}

\section{Subjects}

This was a prospective cohort study. Our inclusion criteria were: 1) patients priorly diagnosed with autoimmune liver disease; 2 ) age of 20 years old or more; 3) patients with no prior knowledge regarding, and no updated information on COVID-19 and the vaccines for SARS-CoV-2 that we used in this study; and 4) patients who consented to participate in this study. Between March 2021 and May 2021, all patients who had a prior diagnosis of either AlH, PBC, PSC, or overlap syndrome were asked to join this study. All information regarding COVID-19 and vaccines in patients with autoimmune liver disease were presented on the website of the interactable hepato-biliary disease study group (http://www.hepatobiliary.jp/). Following ballets were summary of the information.

- About COVID-19

COVID-19 in patients with liver disease

- About novel coronavirus vaccine

Efficacy to patients with autoimmune liver disease

Adverse effect to patients with autoimmune liver disease

- Current recommendation about vaccination for SARS-CoV-2 to patients with autoimmune liver disease 
Thisstudy protocol was approved by the Institutional Review Board of Iwate Medical University (MH2021020) and was registered in the University Hospital Medical Information Network (UMIN000044128). Part of this study's results, comprising 40/71 patients included, has been previously published elsewhere. The study was performed in accordance with the relevant guidelines and regulations, and informed consent was obtained from all participants.

\section{Questionnaire for anxiety about vaccines for SARS-CoV-2}

We used a questionnaire to assess anxiety regarding vaccines, and one was a visual analog scale (VAS) before information-sharing. To evaluate anxiety using the VAS, the left side of a $100 \mathrm{~mm}$ bar represented the strongest anxiety with the greatest extent at $0 \mathrm{~mm}$, while the right side represented no anxiety with the greatest extent at $100 \mathrm{~mm}$. The middle section, representing neither, was located at $50 \mathrm{~mm}$ (Figure1). Before reading the provided description of the updated vaccine information, each participant was asked whether they had read the description before. To assess the effect of providing updated information about COVID-19 and its vaccines, the participant answered the same questions after reading the information.

\section{Data collection}

Age, sex, diagnosis, disease severity (chronic hepatitis or live cirrhosis), drug history, and laboratory data were also collected alongside the questionnaire. Blood cell counts, plasma prothrombin time, and serum levels of alanine aminotransferase, aspartate aminotransferase, creatinine, and total bilirubin were analyzed using an autoanalyzer (JCA-BM2250; JEOL, Tokyo, Japan).

\section{Statistical analysis}

The results were expressed as median and range. All statistical analyses were performed using the SPSS 17.0 software program (SPSS Inc., Chicago, IL, United States). Correlation among continuous values was evaluated using Spearman's correlation. Wilcoxon signed-rank, McNemar, Mann-Whitney and KruskalWallis tests were used to evaluate the statistical significance of the results. A two-sided $p$-value of $<0.05$ was considered statistically significant.

\section{Sample sizes}

To design this study's research protocol, we calculated the required sample size for the primary aim. To assess the effect of information-sharing about COVID-19 and its vaccines on anxiety in patients with autoimmune liver disease, we hypothesized a change of $20 \mathrm{~mm}$ on the VAS from providing information and a $30 \mathrm{~mm}$ standard deviation of change, according to a previous study about anxiety in the perioperative period ${ }^{33}$. The minimum sample size was calculated as 20 patients using an alpha error of 0.05 and a power of 0.8 in paired samples. 


\section{Results}

\section{Patients' characteristics}

Seventy-one consecutive out-patients with autoimmune liver diseases were asked to join this study. All patients agreed to participate in this study. After assessing them for the inclusion criteria, we found that no patients had previously read the descriptions about COVID-19 and its vaccines, and thus all were eligible to participate in the present study. Therefore, the required sample size we had calculated prior to the study was met. The numbers of patients with each etiology of autoimmune liver disease were: 30 with AlH, 30 with PBC, seven with overlap syndrome, and four with PSC (Table 1). According to the severity of liver disease, $86 \%$ of patients had chronic hepatitis because ten cirrhotic patients were included. Of the 71 patients, $85 \%$ were female. No patient had overt hepatic encephalopathy or jaundice. Laboratory data were summarized in Table 1. 
Table 1

Patients' characteristic

\begin{tabular}{|c|c|c|c|c|}
\hline \multicolumn{5}{|l|}{ Etiology } \\
\hline & \multicolumn{2}{|c|}{ Autoimmune hepatitis } & \multicolumn{2}{|l|}{30} \\
\hline & \multicolumn{2}{|c|}{ Primary Biliary cholangitis } & \multicolumn{2}{|l|}{30} \\
\hline & \multicolumn{2}{|c|}{ Overlap syndrome } & \multicolumn{2}{|l|}{7} \\
\hline & \multicolumn{2}{|c|}{ Primary sclerosing cholangitis } & \multicolumn{2}{|l|}{4} \\
\hline Severity & \multicolumn{2}{|c|}{ Chronic hepatitis: liver cirrhosis } & \multicolumn{2}{|l|}{$61: 10$} \\
\hline Sex & \multicolumn{2}{|c|}{ Female } & \multicolumn{2}{|l|}{60} \\
\hline Age & & Years & 61 & $(21-81)$ \\
\hline \multirow[t]{3}{*}{ Medication } & \multicolumn{2}{|c|}{ Ursodeoxycholic acid (+) : (-) } & \multicolumn{2}{|l|}{$45: 26$} \\
\hline & \multicolumn{2}{|c|}{ Prednisolone (+) : (-) } & \multicolumn{2}{|l|}{$35: 36$} \\
\hline & \multicolumn{2}{|c|}{ Azathioprine } & \multicolumn{2}{|l|}{$14: 54$} \\
\hline \multicolumn{3}{|c|}{ Laboratory data } & Median & Range \\
\hline & Alb & $\mathrm{mg} / \mathrm{dL}$ & 4.0 & $(2.1-4.6)$ \\
\hline & T-Bil & $\mathrm{mg} / \mathrm{dL}$ & 0.8 & $(0.2-2.5)$ \\
\hline & AST & $\mathrm{U} / \mathrm{L}$ & 27 & $(12-99)$ \\
\hline & ALT & $\mathrm{U} / \mathrm{L}$ & 25 & $(8-149)$ \\
\hline & gGT & $\mathrm{U} / \mathrm{L}$ & 46 & $(12-449)$ \\
\hline & ALP & $\mathrm{U} / \mathrm{L}$ & 85 & $(30-361)$ \\
\hline & Cre & $\mathrm{mg} / \mathrm{dL}$ & 0.70 & $(0.20-1.34)$ \\
\hline & Plt & $10^{3} / \mathrm{mL}$ & 225 & $(62-548)$ \\
\hline & PT-IN & & 1.00 & $(0.91-1.12)$ \\
\hline
\end{tabular}

Provision of information regarding COVID-19 and its vaccine supported self-decision for vaccination and reduced anxiety toward the vaccine

Before information-sharing of the description of COVID-19 and its vaccines, the number of patients who would receive vaccination was 50 , while $23 \%(16 / 71)$ of patients could not decide on receiving the vaccine (Table 2). After information-sharing, the number of patients who could not decide on receiving the vaccine decreased to six ( $8 \%)$. Of the remaining 65 patients, 59 decided to receive the vaccine, while 
six did not. There was a significant difference in the distribution of the answer to "Will you receive the vaccine for SARS-CoV-2?" after information-sharing ( $p=0.00533$, Wilcoxon signed-rank test).

Table 2

Effect of information sharing to answer for questionnaire and VAS for anxiety to vaccine for SARS-CoV-2 in all subjects.

\begin{tabular}{|c|c|c|c|}
\hline Will you receive vaccine for SARS-CoV-2? & Before & After & $\mathrm{p}$ value \\
\hline I will & 50 & 59 & \multirow[t]{3}{*}{${ }^{*} p=0.00533$} \\
\hline I will not & 5 & 6 & \\
\hline Uncertain & 16 & 6 & \\
\hline $\begin{array}{l}\text { Do you feel anxiety to vaccine for SARS-CoV- } \\
2 \text { ? }\end{array}$ & Before & After & $p$ value \\
\hline Yes & 46 & 23 & \multirow{2}{*}{$\begin{array}{l}* \star p= \\
0.0000108\end{array}$} \\
\hline \multirow[t]{2}{*}{ No } & 25 & 48 & \\
\hline & $\begin{array}{l}\text { Median } \\
\text { (Range) }\end{array}$ & $\begin{array}{l}\text { Median } \\
\text { (Range) }\end{array}$ & \\
\hline VAS (mm) & $35(0-100)$ & $72(0-100)$ & ${ }^{*} \mathrm{p}<0.00001$ \\
\hline \multicolumn{4}{|c|}{$\begin{array}{l}\text { Asterisk indicated that data was analyzed using Wilcoxon signed-rank test. Double asterisks indicate } \\
\text { that data was analyzed using McNemar test. }\end{array}$} \\
\hline
\end{tabular}

When evaluating anxiety regarding the vaccine, $65 \%$ of the patients felt anxiety toward the vaccine initially (Table 2). Because the median value was $35 \mathrm{~mm}$ on the VAS, which implied feeling anxiety before receiving the information, the results of the questionnaire and VAS showed similar tendencies. After information-sharing by reading the provided description, only $32 \%$ of the patients felt anxiety regarding the vaccine, thus indicating a significant decrease $(p=0.0000108$, McNemar test). Similarly, a $72 \mathrm{~mm}$ median VAS was noted after information-sharing, indicating a significant improvement $(p<0.00001$, Wilcoxon signed-rank test).

\section{Information-sharing was more effective for patients who felt strong anxiety before receiving information}

To assess the effect of information-sharing on patients' anxiety, the correlation between the change in VAS after providing information (Delta-VAS) and either VAS before- (Pre-VAS) or after receiving the information (Post-VAS) was evaluated (Fig. 2a and 2b). Delta-VAS was calculated as subtracted values between Post-VAS and Pre-VAS responses. Delta-VAS negatively correlated with Pre-VAS (rho $=-0.4050$, $p=0.00046$; Spearman's correlation), and positively correlated with Post-VAS ( $r h o=0.3020, p=0.0104$; Spearman's correlation). Patients who answered with low values on Pre-VAS exhibited reduced anxiety after being provided information, while only a small part of the patients with high values on Post-VAS had answered with high values on Pre-VAS regardless of receiving the information. 
Age, medication, or etiology of autoimmune liver disease were not associated with anxiety or the effect of information-sharing on anxiety

To assess the specific characteristics of patients who felt strong anxiety or required support for selfdecision, we compared the correlations of VAS with age, medication, or etiology of autoimmune liver disease. We evaluated the correlation of patient age with Pre-VAS, Post-VAS, or Delta-VAS (Fig. 3a, 3b, and 3c). There was no significant correlation in any comparison. Because elderly age was known as a risk factor of unfavorable prognosis in patients with COVID-19, subjects were divided into two groups: over 65 years old and less than 65 years old. There was no significant difference regarding the Pre-VAS, Post-VAS, or Delta-VAS between these two groups (Fig. 4). Because autoimmune liver disease was treated using ursodeoxycholic acid (UDCA) and/or prednisolone (PSL), elucidating whether there was a difference in anxiety among patients treated with UDCA and PSL was desired. To assess this, subjects were divided by administration of UDCA and PSL, with Pre-VAS, Post-VAS, or Delta-VAS compared. There was no significant difference between groups with or without UDCA and with or without PSL (Fig. 5a and 5b). Because the etiology of autoimmune liver disease might affect anxiety about the SARS-CoV-2 vaccine, we compared VAS among each etiology. On comparing Pre-VAS, Post-VAS, or Delta-VAS, there were no significant differences associated with any etiology of autoimmune hepatitis (Fig. 6).

\section{Information-sharing was effective in supporting self-decision among AlH patients and reduced anxiety in both AlH and PBC patients}

Because the number of patients with both AlH and PBC satisfied our sample size requirements, the effect of information-sharing for both self-decision and anxiety was evaluated within both $\mathrm{AlH}$ and PBC groups. In the AlH group, providing information increased the number of patients who decided to receive the vaccine and decreased those who could not decide on receiving a vaccination (Table 3. $p=0.0105$, Wilcoxon signed-rank test). Conversely, information-sharing did not significantly change the number of patients able to self-decide on vaccination in the PBC group (Table 4). On evaluating the effect of information-sharing on anxiety regarding vaccines, the number of anxious patients significantly decreased in both AlH and PBC groups after receiving the information (Tables 3 and $4 ; p=0.004427$ for $A I H, p=0.0159$ for PBC, McNemar test). Similarly, VAS for anxiety also improved in both AlH and PBC groups after receiving the information (Tables 3 and 4. $p=0.0000717$ for $\mathrm{AlH}, \mathrm{p}=0.000395$ for PBC, Wilcoxon signed-rank test). 
Table 3

Effect of information sharing to answer for questionnaire and VAS for anxiety to vaccine for SARS-CoV-2 in subjects with autoimmune hepatitis.

\begin{tabular}{|c|c|c|c|}
\hline Will you receive vaccine for SARS-CoV-2? & Before & After & $\mathrm{p}$ value \\
\hline I will & 18 & 25 & \multirow[t]{3}{*}{${ }^{\star} p=0.0105$} \\
\hline I will not & 2 & 2 & \\
\hline Uncertain & 10 & 3 & \\
\hline $\begin{array}{l}\text { Do you feel anxiety to vaccine for SARS-CoV- } \\
\text { 2? }\end{array}$ & Before & After & $\mathrm{p}$ value \\
\hline Yes & 18 & 8 & \multirow[t]{2}{*}{${ }^{* *} p=0.004427$} \\
\hline \multirow[t]{2}{*}{ No } & 12 & 22 & \\
\hline & $\begin{array}{l}\text { Median } \\
\text { (Range) }\end{array}$ & $\begin{array}{l}\text { Median } \\
\text { (Range) }\end{array}$ & \\
\hline VAS (mm) & $35(0-100)$ & $74(20-100)$ & $\begin{array}{l}* \mathrm{p} p= \\
0.0000717\end{array}$ \\
\hline \multicolumn{4}{|c|}{$\begin{array}{l}\text { Asterisk indicated that data was analyzed using Wilcoxon signed-rank test. Double asterisks indicatec } \\
\text { that data was analyzed using McNemar test. }\end{array}$} \\
\hline VAS, Visual Analog Scale & & & \\
\hline
\end{tabular}


Table 4

Effect of information sharing to answer for questionnaire and VAS for anxiety to vaccine for SARS-CoV-2 in subjects with primary biliary cholangitis.

\begin{tabular}{|c|c|c|c|}
\hline Will you receive vaccine for SARS-CoV-2? & Before & After & $\mathrm{p}$ value \\
\hline I will & 23 & 23 & \multirow[t]{3}{*}{$*_{\text {n.s. }}$} \\
\hline I will not & 3 & 4 & \\
\hline Uncertain & 4 & 3 & \\
\hline $\begin{array}{l}\text { Do you feel anxiety to vaccine for SARS-CoV- } \\
\text { 2? }\end{array}$ & Before & After & $\mathrm{p}$ value \\
\hline Yes & 18 & 9 & \multirow[t]{2}{*}{$* \star p=0.0159$} \\
\hline \multirow[t]{2}{*}{ No } & 12 & 21 & \\
\hline & $\begin{array}{l}\text { Median } \\
\text { (Range) }\end{array}$ & $\begin{array}{l}\text { Median } \\
\text { (Range) }\end{array}$ & \\
\hline VAS (mm) & $46(0-95)$ & $72(0-100)$ & $\begin{array}{l}{ }^{*} p= \\
0.000395\end{array}$ \\
\hline \multicolumn{4}{|c|}{$\begin{array}{l}\text { Asterisk indicated that data was analyzed using Wilcoxon signed-rank test. Double asterisks indicatec } \\
\text { that data was analyzed using McNemar test. }\end{array}$} \\
\hline \multicolumn{4}{|l|}{ VAS, Visual Analog Scale } \\
\hline
\end{tabular}

\section{Discussion}

The present study demonstrated that: 1) patients with autoimmune liver disease felt anxiety towards the SARS-CoV-2 vaccine; 2) information-sharing via a description with updated information reduced anxiety regarding the vaccine; 3 ) information-sharing encouraged patients to decide favorably toward receiving the vaccine; and 4) a beneficial effect towards improving anxiety was found in patients with strong anxiety before being provided the information.

Over 150 million people have been infected, and over 3 million have died due to COVID-19, according to a report from the world health organization on May 20, 2021 ${ }^{15}$. Vaccination prevents progression to severe grades of COVID-19 16,17; therefore, vaccines to prevent SARS-CoV-2 infection are considered the most promising approach for curbing the pandemic. In Japan, elderly citizens and patients with chronic diseases started to receive the vaccine on May $2021^{18}$. Generally, infectious disease management is classified into three stages: Control, Elimination, and Eradication ${ }^{19}$. To achieve Control or Eradication of infectious disease, a sufficient number of patients must receive effective vaccination ${ }^{8}$; therefore, as many people as possible need to receive vaccines for SARS-CoV-2 to control the COVID-19 pandemic. However, some do not receive the vaccines because they fear their side effects ${ }^{9}$. In this study, patients with autoimmune liver disease felt anxiety about the vaccine. 
Further, there were no unifying characteristics of these patients who felt anxiety. Because Delta-VAS negatively correlated with Pre-VAS, information-sharing was more effective in patients with strong anxiety before receiving the information. Additionally, the number of patients who did not opt to receive vaccines decreased after being provided the information. Consequently, information-sharing with patients from physicians encouraged self-decision for receiving the vaccine and was important for increasing the number who opted to receive the vaccine.

Patients after transplantation who were treated with azathioprine revealed a low response rate to the vaccine for SARS-CoV- $2^{20}$. Although it is unknown that azathioprine may affect responses to the vaccine in patients with autoimmune liver disease, some of the patients treated with azathioprine needed to be carefully observed after vaccination. In addition, some infections in fully vaccinated individuals have been reported ${ }^{21}$. Because COVID-19 will be a precipitating event to acute-onset chronic liver failure in patients with chronic hepatitis ${ }^{22}$, COVID-19 infection in patients with chronic hepatitis should be prevented as much as possible. Personal preventive measures have still been encouraged to this population to prevent SARS-CoV-2 infection via reduction of transmission.

Because social media are widely used, patients may receive an overflow of information from these sources. However, some of the information within social media is presented without scientific inspection ${ }^{23}$. When patients are provided contradicting information, they are unable to make adequately informed decisions. Conversely, patients can make decisions to solve their health problems when a physician appropriately provides information based on scientific evidence ${ }^{24}$. Therefore, physicians must recognize two essential steps for controlling COVID-19: first, physicians must collect data about vaccines' beneficial or adverse effects in patients with autoimmune liver disease; second, physicians must communicate scientifically-based information with their patients to support patients' self-decision regarding vaccination. In this study, the specific characteristics of patients who felt strong anxiety toward the vaccine were not identified. Thus, we need to understand that all patients may feel anxiety towards the vaccine. Moreover, the way in which information-sharing affects potentially anxious patients is important, because no participant in this study had read this information prior to this study.

According to previous reports on vaccines for viral infections, several factors, such as race, age, and comorbidity, are associated with the duration of sufficient levels of neutralized antibodies, response to vaccines, or frequency of long-term effects of vaccination ${ }^{25-29}$. Currently, recently established vaccines for SARS-CoV-2 require universal vaccination to control disease spread ${ }^{30}$. How long the efficacy of vaccination continues remains unknown, although several papers have reported antibody levels for SARS-CoV-2 in patients who had COVID-19 or received the vaccine ${ }^{31,32}$. Additionally, whether the second dose of vaccine would be acceptable to patients who show adverse effects after the first and whether a different vaccine for the second dose would be effective needs to be discussed. To establish a schedule for vaccination, the chronological change in antibody level, the response rate to vaccination, or sufficient level of antibody production to prevent infection needs to be researched in future studies. These 
considerations need to be solved in patients with autoimmune liver disease as well as the general population.

There are several limitations in this study. Firstly, the participants had never read the provided information before the study. Further intervention to increase the number of patients who read the description needs to be considered. Secondly, the number of patients with cirrhosis was small. Thus, whether informationsharing can reduce anxiety in patients with a high risk of death due to COVID-19 remains unknown. Finally, whether physicians recognize patients' anxiety remains unknown. Because information-sharing by physicians helped patients understand the importance of vaccination, the provision of information should be the first step. We hope the present study may let physicians know that information-sharing can increase the number of patients who receive vaccination via reduced anxiety about the SARS-CoV-2 vaccine.

The present study has presented that information-sharing can decrease anxiety to vaccination for COVID19 in patients with autoimmune liver disaes. Information of vaccination in specific conditions such as immune suppresive state needs to be appropriately provided to patients when patients with autoimmune liver diasee feel anxiety to the vaccination to SARS-CoV-2.

Herein, we have reported that subjects with autoimmune liver disease feel anxiety toward the COVID-19 vaccine, and appropriate information-sharing about the vaccine for SARS-CoV-2 reduces this anxiety, resulting in the subjects' deciding to undergo vaccination. Information of vaccination to COVID-19 in specific conditions such as immune suppresive state needs to be appropriately provided to patients.

\section{Declarations}

\section{Funding information}

This work was supported by Japanese Ministry of Health, Labour and Welfare Research Program on Intractable Hepatobiliary Disease, Grant Number JPMH20FC1023.

\section{Ethics approval}

This study protocol was approved by the institutional ethical committee (MH2021-020) and was registered in the University Hospital Medical Information Network (UMIN000044128).

\section{Consent to participate}

Informed consent was obtained from all participants in this study.

\section{Author contribution}

KK: Design of the research, analysis, drafting the manuscript, final approval of the article

YS: Acquisition of data, final approval of the article 
HA: Analysis, final approval of the article

HE: Acquisition of data, final approval of the article

KY: Acquisition of data, final approval of the article

YT: drafting the manuscript, final approval of the article

\section{References}

1. Wiersinga, W. J., Rhodes, A., Cheng, A. C., Peacock, S. J. \& Prescott, H. C. Pathophysiology, Transmission, Diagnosis, and Treatment of Coronavirus Disease 2019 (COVID-19): A Review. JAMA 324, 782-793, doi:10.1001/jama.2020.12839 (2020).

2. Satapathy, S. K. et al. Risk factors and outcomes for acute-on-chronic liver failure in COVID-19: a large multi-center observational cohort study. Hepatol Int, doi:10.1007/s12072-021-10181-y (2021).

3. Oh, H. J. \& Lee, H. When Do People Verify and Share Health Rumors on Social Media? The Effects of Message Importance, Health Anxiety, and Health Literacy. J Health Commun 24, 837-847, doi:10.1080/10810730. 2019.1677824 (2019).

4. Tan, S. S. Goonawardene, N. Internet Health Information Seeking and the Patient-Physician Relationship: A Systematic Review. J Med Internet Res 19, e9, doi:10.2196/jmir.5729 (2017).

5. Rottem, M. \& Shoenfeld, Y. Vaccination and allergy. Curr Opin Otolaryngol Head Neck Surg 12, 223231, doi:10.1097/01.moo.0000122312.13359.eb (2004).

6. Schwartz, K. L. et al. Racial similarities in response to standardized offer of influenza vaccination. A MetroNet study. J Gen Intern Med 21, 346-351, doi:10.1111/j.1525-1497.2006.00401.x (2006).

7. Sakai, A. et al. Identification of amino acids in antigen-binding site of class II HLA proteins independently associated with hepatitis $B$ vaccine response. Vaccine $35,703-710$, doi:10.1016/j.vaccine.2016.08.068 (2017).

8. Kakisaka, K. et al. Hepatitis B Surface Antibody Titers at One and Two Years after Hepatitis B Virus Vaccination in Healthy Young Japanese Adults. Intern Med 58, 2349-2355, doi:10.2169/internalmedicine.2231-18 (2019).

9. Nishida, N. et al. Importance of HBsAg recognition by HLA molecules as revealed by responsiveness to different hepatitis B vaccines. Sci Rep 11, 3703, doi:10.1038/s41598-021-82986-8 (2021).

10. https://www.cdc.gov/coronavirus/2019-ncov/vaccines/vaccine-benefits.html. Accessed on May 20. ( 2021).

11. Chia, W. N. et al. Dynamics of SARS-CoV-2 neutralising antibody responses and duration of immunity: a longitudinal study. Lancet Microbe, doi:10.1016/S2666-5247(21)00025-2 (2021).

12. Ogata, A. F. et al. Ultra-sensitive Serial Profiling of SARS-CoV-2 Antigens and Antibodies in Plasma to Understand Disease Progression in COVID-19 Patients with Severe Disease. Clin Chem, doi:10.1093/clinchem/hvaa213 (2020). 
13. Elkins, G., Staniunas, R., Rajab, M. H. \& Marcus, J. Snyder, T. Use of a numeric visual analog anxiety scale among patients undergoing colorectal surgery. Clin Nurs Res 13, 237-244, doi:10.1177/1054773803262222 (2004).

14. 13. Beuers, U. \& Rust, C. Overlap syndromes. Semin Liver Dis 25, 311-320, doi:10.1055/s-2005916322 (2005).

15. 14. Cornberg, M., Buti, M., Eberhardt, C. S., Grossi, P. A. \& Shouval, D. EASL position paper on the use of COVID-19 vaccines in patients with chronic liver diseases, hepatobiliary cancer and liver transplant recipients. J Hepatol 74, 944-951, doi:10.1016/j.jhep.2021.01.032 (2021).

16. 15. https://covid19.who.int/. Accessed on May 20. (2021).

17. 16. Polack, F. P. et al. Safety and Efficacy of the BNT162b2 mRNA Covid-19 Vaccine. N Engl J Med 383, 2603-2615, doi:10.1056/NEJMoa2034577 (2020).

18. 17. Voysey, M. et al. Safety and efficacy of the ChAdOx1 nCoV-19 vaccine (AZD1222) against SARSCoV-2: an interim analysis of four randomised controlled trials in Brazil, South Africa, and the UK. Lancet 397, 99-111, doi:10.1016/S0140-6736(20)32661-1 (2021).

19. 18. https://www.mhlw.go.jp/stf/seisakunitsuite/bunya/0000121431_00218.html. Accesed on May 20 (2021).

20. 19. Dowdle, W. R. The principles of disease elimination and eradication. Bull World Health Organ 76 Suppl 2, 22-25 (1998).

21. 20. Boyarsky, B. J. et al. Antibody Response to 2-Dose SARS-CoV-2 mRNA Vaccine Series in Solid Organ Transplant Recipients. JAMA, doi:10.1001/jama.2021.7489 (2021).

22. 21. https://www.cdc.gov/vaccines/covid-19/health-departments/breakthrough-cases.html. Accessed on May 4, <https://www.cdc.gov/vaccines/covid-19/health-departments/breakthrough-cases.html> (2021).

23. 22. Satapathy, S. K. et al. Risk factors and outcomes for acute-on-chronic liver failure in COVID-19: a large multi-center observational cohort study. Hepatol Int, doi:10.1007/s12072-021-10181-y (2021).

24. 23. Oh, H. J. \& Lee, H. When Do People Verify and Share Health Rumors on Social Media? The Effects of Message Importance, Health Anxiety, and Health Literacy. J Health Commun 24, 837-847, doi:10.1080/10810730.2019.1677824 (2019).

25. 24. Tan, S. S. \& Goonawardene, N. Internet Health Information Seeking and the Patient-Physician Relationship: A Systematic Review. J Med Internet Res 19, e9, doi:10.2196/jmir.5729 (2017).

26. 25. Rottem, M. \& Shoenfeld, Y. Vaccination and allergy. Curr Opin Otolaryngol Head Neck Surg 12 , 223-231, doi:10.1097/01.moo.0000122312.13359.eb (2004).

27. 26. Schwartz, K. L. et al. Racial similarities in response to standardized offer of influenza vaccination. A MetroNet study. J Gen Intern Med 21, 346-351, doi:10.1111/j.15251497.2006.00401.x (2006).

28. 27. Sakai, A. et al. Identification of amino acids in antigen-binding site of class II HLA proteins independently associated with hepatitis B vaccine response. Vaccine 35, 703-710, 
doi:10.1016/j.vaccine.2016.08.068 (2017).

29. 28. Kakisaka, K. et al. Hepatitis B Surface Antibody Titers at One and Two Years after Hepatitis B Virus Vaccination in Healthy Young Japanese Adults. Intern Med 58, 2349-2355, doi:10.2169/internalmedicine.2231-18 (2019).

30. 29. Nishida, N. et al. Importance of HBsAg recognition by HLA molecules as revealed by responsiveness to different hepatitis B vaccines. Sci Rep 11, 3703, doi:10.1038/s41598-021-82986-8 (2021).

31. 30. https://www.cdc.gov/coronavirus/2019-ncov/vaccines/vaccine-benefits.html. Accessed on May 20. (2021).

32. 31. Chia, W. N. et al. Dynamics of SARS-CoV-2 neutralising antibody responses and duration of immunity: a longitudinal study. Lancet Microbe, doi:10.1016/S2666-5247(21)00025-2 (2021).

33. 32. Ogata, A. F. et al. Ultra-sensitive Serial Profiling of SARS-CoV-2 Antigens and Antibodies in Plasma to Understand Disease Progression in COVID-19 Patients with Severe Disease. Clin Chem, doi:10.1093/clinchem/hvaa213 (2020).

34. 33. Elkins, G., Staniunas, R., Rajab, M. H., Marcus, J. \& Snyder, T. Use of a numeric visual analog anxiety scale among patients undergoing colorectal surgery. Clin Nurs Res 13, 237-244, doi:10.1177/1054773803262222 (2004).

\section{Figures}

Figure1

1. Will you receive vaccine for SARS-CoV-2?

口 I will

ㅁ I will not

ㅁ Uncertain

2. Do you feel anxiety to vaccine for SARS-CoV-2?

口 Yes

№

3. Please describe your anxiety to vaccine for SARS-CoV-2.

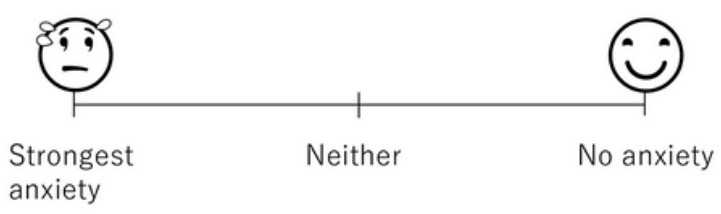


Questionnaire in this study.

Figure 2

a

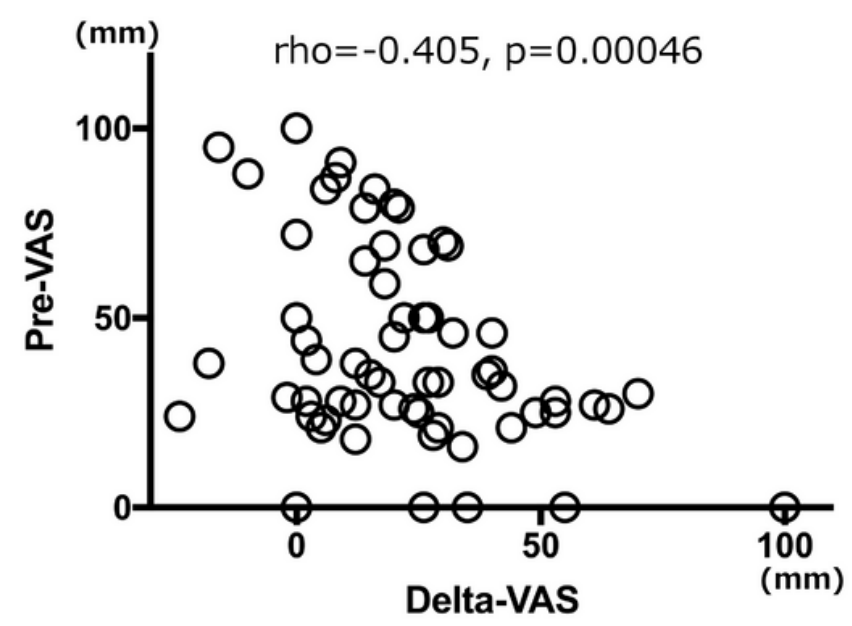

b

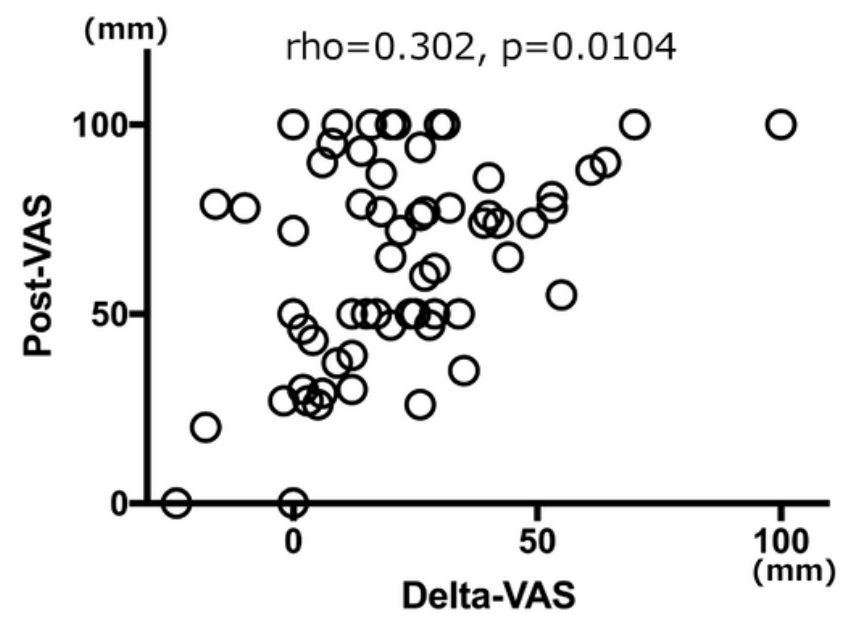

Spearman's correlation

\section{Figure 2}

Effect of information-sharing on anxiety regarding COVID-19 and the SARS-CoV-2 vaccines To assess information-sharing's effect on patient anxiety, a correlation using a visual analog scale (VAS) scores before/after information-sharing (Pre-VAS/Post-VAS) and the change in VAS scores after informationsharing (Delta-VAS) were evaluated. The vertical axis indicates Pre-VAS (Fig. 2-a) or Post-VAS (Fig. 2-b) scores. The horizontal axis indicates the Delta-VAS. Correlations were analyzed using Spearman's correlation. 
Figure3

a $(\mathrm{mm})$

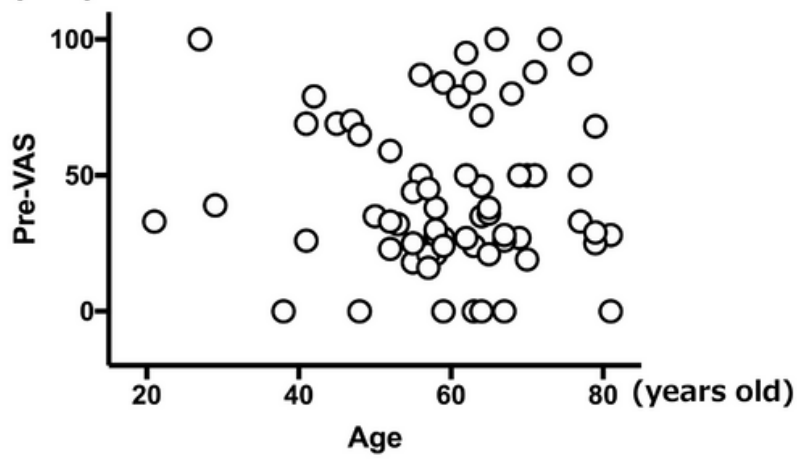

C

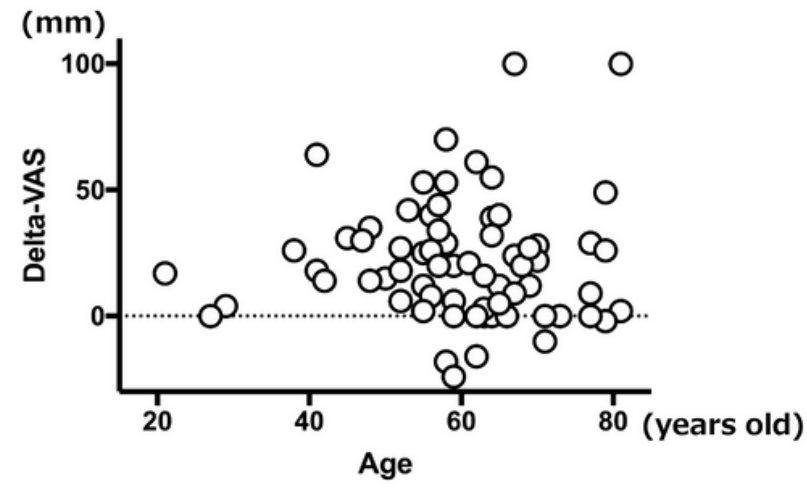

b $\quad(\mathrm{mm})$

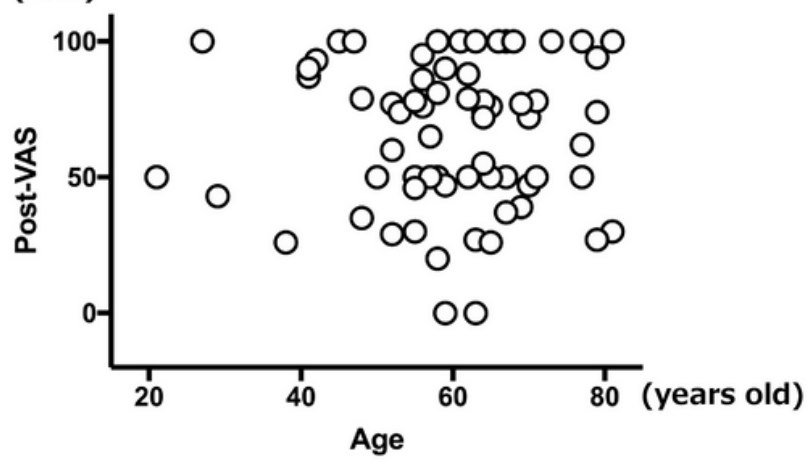

\section{Figure 3}

Correlation between age and anxiety towards the SARS-CoV-2 vaccine Correlation between age and visual analog scale (VAS) scores before/after information-sharing (Pre-VAS/Post-VAS) or the change in VAS scores after information-sharing (Delta-VAS) were evaluated. The vertical axis indicates Pre-VAS (Fig. 3a), Post-VAS (Fig. 3-b), or Delta-VAS (Fig. 3-c) scores. The horizontal axis indicates age. The correlation was analyzed using Spearman's correlation. 
Figure 4

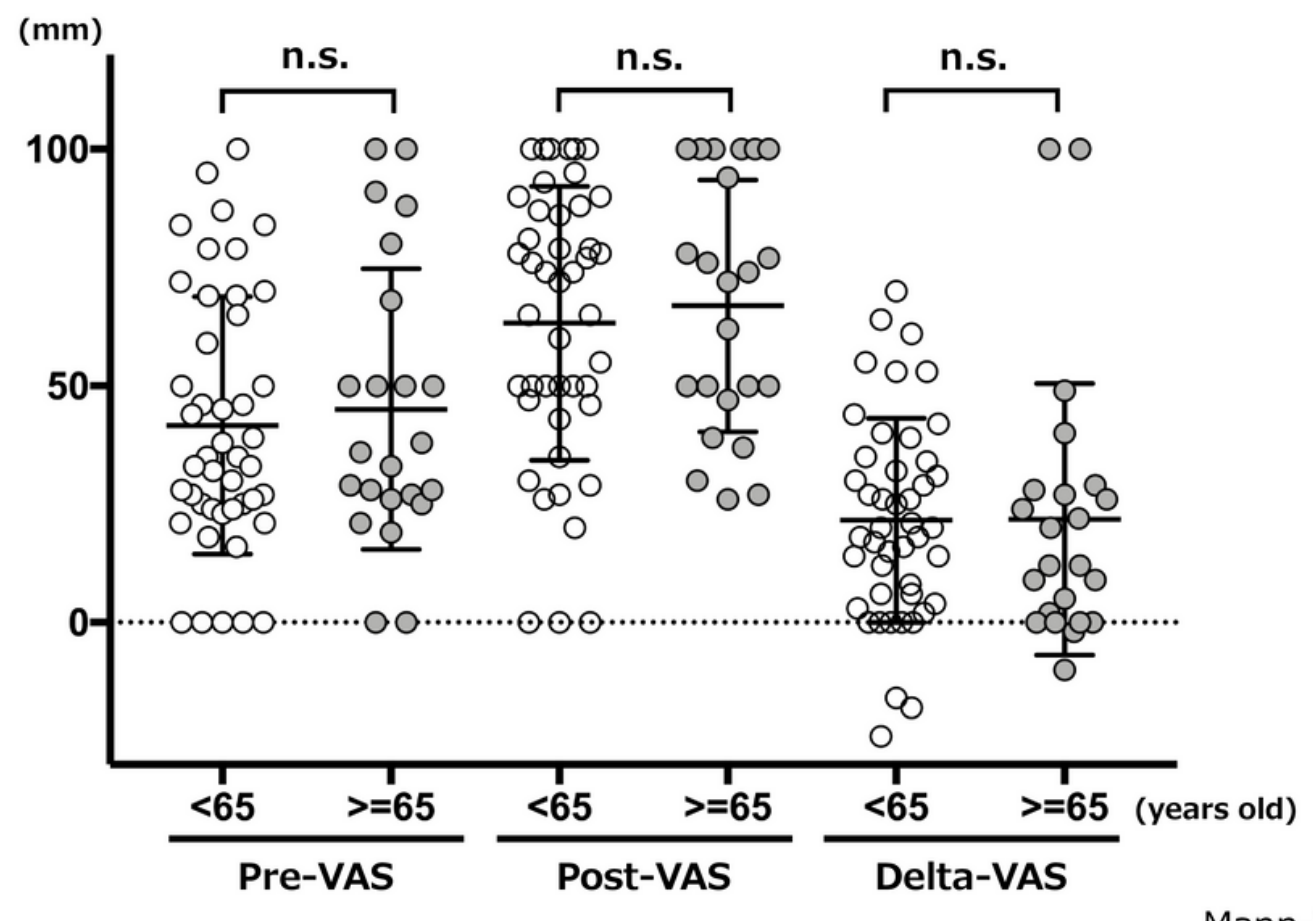

Mann-Whitney U test

\section{Figure 4}

Comparison of anxiety towards SARS-CoV-2 vaccine between non-elderly (less than 65 years old) and elderly (65 years old over) groups Visual analog scale (VAS) scores before/after information-sharing (PreVAS/Post-VAS) or change of VAS scores after information-sharing (Delta-VAS) were compared between non-elder (less than 65 years old: $<65$ ) and elder $(65$ years old over: > $=65)$ groups. The vertical axis indicates Pre-VAS, Post-VAS, or Delta-VAS scores. The horizontal axis indicates each comparison. The difference between groups was analyzed using the Mann-Whitney $U$ test. P-value $<0.05$ was considered as a statistically significant difference. 
Figure5

a

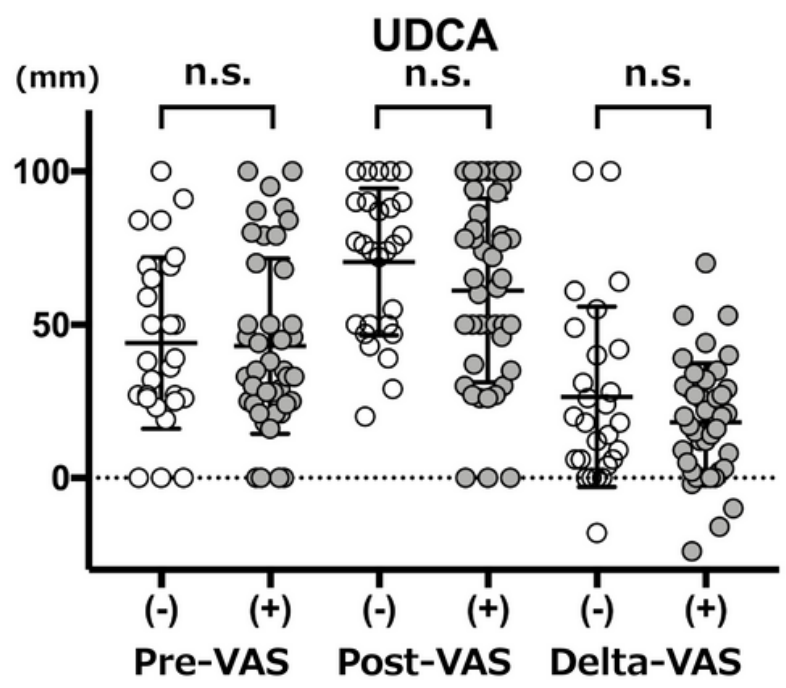

b

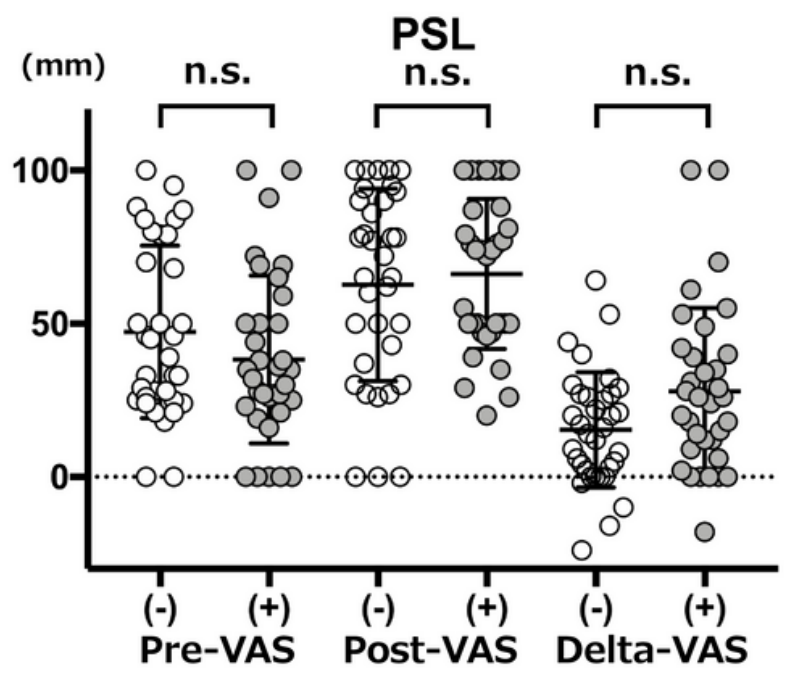

Mann-Whitney U test

\section{Figure 5}

Comparison of anxiety towards SARS-CoV-2 vaccine between patients with/without ursodeoxycholic acid or prednisolone use. Visual analog scale (VAS) scores before/after information-sharing (Pre-VAS/PostVAS) or change of VAS scores after information-sharing (Delta-VAS) were compared between patients with/without ursodeoxycholic acid or prednisolone use. The vertical axis indicates Pre-VAS, Post-VAS, or Delta-VAS. The horizontal axis indicates each comparison. The difference between groups was analyzed using the Mann-Whitney U test. P-value $<0.05$ was considered as a statistically significant difference. 
Figure 6

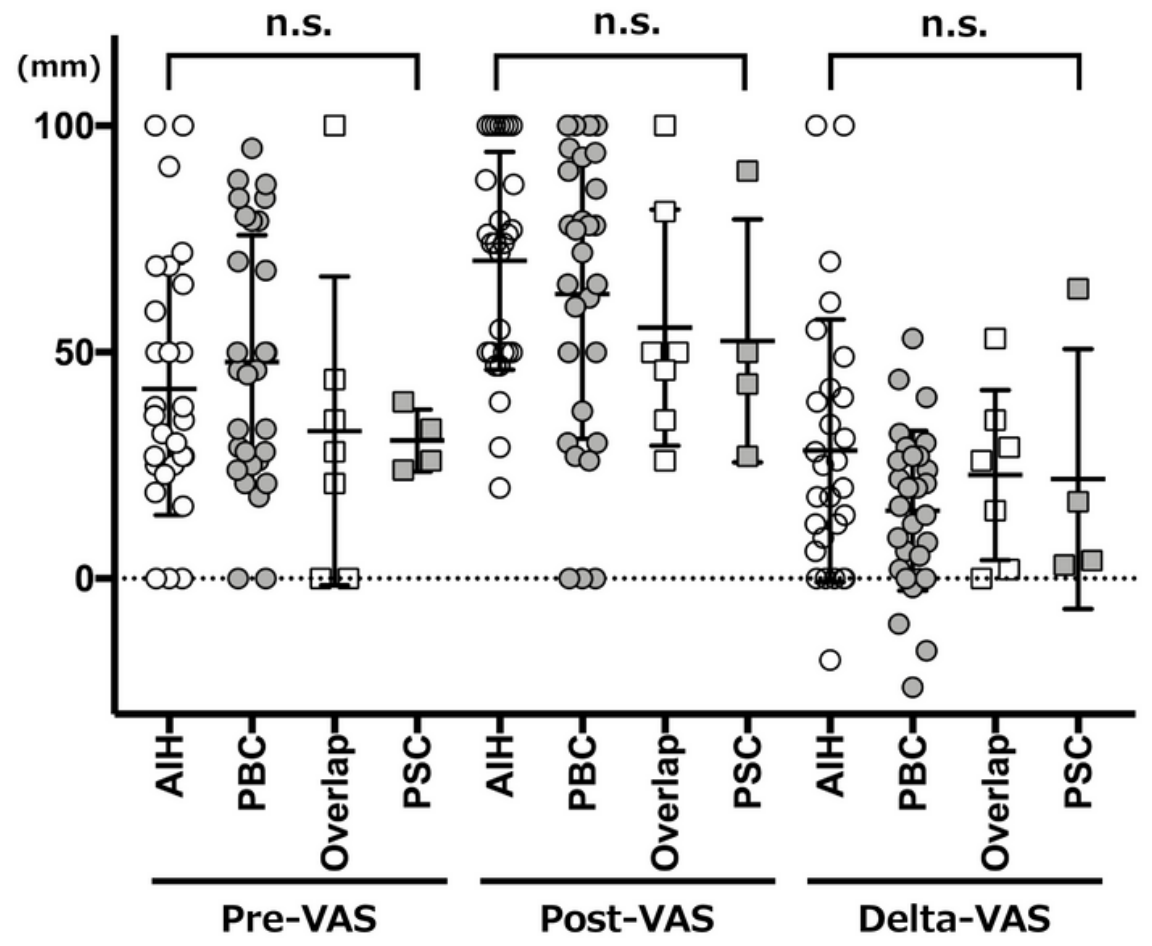

Kruskal-Wallis test

\section{Figure 6}

Comparison of anxiety towards SARS-CoV-2 vaccine among patients with different etiologies of autoimmune liver disease. Visual analog scale (VAS) scores before/after information-sharing (PreVAS/Post-VAS) or change of VAS scores after information-sharing (Delta-VAS) were compared between patients with different etiologies of autoimmune liver disease (autoimmune hepatitis, primary biliary cholangitis, overlap syndrome (Overlap), or primary sclerosing cholangitis). The vertical axis indicates Pre-VAS, Post-VAS, or Delta-VAS. The horizontal axis indicates each comparison. The difference between etiologies was analyzed using the Kruskal-Wallis test. P-value $<0.05$ was considered as a statistically significant difference. 